\title{
Necrólisis epidérmica tóxica con buena respuesta a pulsos de metilprednisolona
}

\section{Toxic epidermal necrolysis with good response to methylprednisolone pulses}

Stalin Vilchez-Rivera ', María Belén Talledo-Serquén ${ }^{2}$, Rodolfo Marcelino Cruz-Villalobos ${ }^{3}$, César Edmundo Vélez-López ${ }^{4}$, Carlos Geraldo Fernández-Narváez ${ }^{4}$, Daniel Manay-Guadalupe ${ }^{4}$
Vílchez-Rivera S, Talledo-Serquén M, Cruz-Villalobos R, Vélez-López C, Fernández-Narváez C, Manay-Guadalupe D. Necrólisis epidérmica tóxica con buena respuesta a pulsos de metilprednisolona. Rev Soc Peru Med Interna. 2020;33(2):77-8I.

https://doi.org//0.36393/spmi.v33i2.524

\section{RESUMEN \\ Mujer de 15 años de edad, que después de recibir lamotrigina por epilepsia no controlada, desarrolló eritema generalizado, ampollas y descamación, con compromiso de mucosas y que abarcó del $60 \%-80 \%$ de la superficie corporal. El cuadro clínico de necrólisis epidérmica tóxica fue confirmado por biopsia de piel.Aparte de las medidas de soporte, se le administró pulsos de metilprednisolona con rápida respuesta favorable.}

Palabras claves: Necrólisis epidérmica tóxica, lamotrigina, metilprednisolona.

\section{ABSTRACT}

A 15-year-old woman, who, after receiving lamotrigine by uncontrolled epilepsy, developed generalized erythema, blisters and flaking, with mucous membrane involvement and covering $60 \%-80 \%$ of the body surface area. The clinical picture of toxic epidermal necrolysis was confirmed by skin biopsy. In addition to support measures, she was given methylprednisolone pulses with rapid favorable response.

Keywords: Toxic epidermal necrolysis, lamotrigine, methylprednisolone.

\section{INTRODUCCIÓN}

La necrólisis epidérmica tóxica (NET) es una emergencia dérmica y constituye gran mortalidad por la severidad al presentar insuficiencia cutánea aguda por comprometer

I. Médico internista. Facultad Medicina Humana. Universidad Nacional Pedro Ruiz Gallo. Servicio de Emergencia, Hospital Regional Lambayeque, Chiclayo.

2. Estudiante de medicina humana. Universidad San Martín de Porras, Chiclayo.

3. Médico intensivista. Facultad Medicina Humana. Universidad Nacional Pedro Ruiz Gallo. Servicio Emergencia. Hospital Regional Lambayeque. Chiclayo.

4. Médico internista. Servicio de Emergencia, Hospital Regional Lambayeque, Chiclayo. gran porcentaje de piel.

La NET es una enfermedad muy rara y grave, se caracteriza por daño a las uniones dermoepidérmicas, necrosis y desprendimiento de la epidermis con compromiso sistémico y forma parte del grupo de enfermedades ampollosas. ${ }^{1,2}$

La NET se genera por una respuesta inmune inapropiada a ciertos fármacos o sus metabolitos. Los fármacos que con mayor frecuencia se relacionan con NET son sulfamidas, pirazolonas, anticonvulsivantes y alopurinol. ${ }^{1,2}$ Dentro de los anticonvulsivantes, los que más destacan son la fenitoína, lamotrigina, carbamazepina y fenobarbital. ${ }^{3}$ El paracetamol e ibuprofeno son fármacos cuya asociación con la enfermedad es poca clara y generan confusión ya que son utilizados en la fase prodrómica pero hay estudios que indican que pueden causar SSJ/NET. Los fármacos contra el cáncer también son causantes. ${ }^{4}$ Las infecciones por Mycoplasma pneumoniae y citomegalovirus son causas comunes, especialmente en niños. ${ }^{5}$ También se han reportado casos tras la vacunación contra el sarampión, parotiditis y rubéola. Pero, es la hipersensibilidad a fármacos la causa principal. ${ }^{2}$

La NET afecta a más del $30 \%$ de superficie corporal y SSJ las lesiones representan menos del 10\%; y, se consideran casos superpuestos a aquellos pacientes con compromiso del 10\% a 30\% de superficie corporal. ${ }^{1}$ La NET también se 
relaciona con estados de inmunosupresión como trasplante de médula ósea, infección por VIH, conectivopatías y malignidad. ${ }^{2}$

La NET se manifiesta como un exantema eritematoso, simétrico y que confluye, que se inicia en la cara y se expande rápidamente por todo el tronco y las extremidades. Es frecuente el compromiso de mucosas, incluyendo la orofaríngea, ocular y genital. El daño a mucosas aparece en el $90 \%$ a $95 \%$ de los casos, usualmente están afectados más de dos mucosas, siendo rara la afectación de una sola. 1,2,5 Las lesiones en piel incluyen maculas eritematosas que pueden estar o no infiltradas. ${ }^{6}$ Las ampollas en la cavidad oral se convierten en erosiones que son muy dolorosas y hemorrágicas, se recubren por una pseudomembrana blanca grisácea que dificulta la alimentación y genera hipersalivación; el compromiso a la mucosa genital con frecuencia aparece en el glande, vulva y vagina y genera ardor al momento de miccionar, retención urinaria y sinequias. Cuando se afecta la conjuntiva aparece fotofobia, dolor, quemosis, y si el daño es muy grave aparecen ulceras corneales, uveítis y puede llegar a la ceguera. ${ }^{7}$

El desprendimiento de la epidermis genera áreas con piel desepitelizada que semeja una quemadura de segundo grado. La dermis denudada segrega suero, se infecta lo que puede causar infecciones sistémicas y además sangra con facilidad. Las ampollas que aparecen son flácidas y existe EL Signo de Nikolsky positivo. ${ }^{1,4}$

La NET tiene TRES fases: la fase prodrómica, el daño cutáneo aparece tras un cuadro de fiebre, tos, anorexia, malestar general que con frecuencia aparece de una a tres semanas después de la ingestión del fármaco sospechoso; el periodo de necrólisis, aparece súbitamente un exantema doloroso, al inicio en cara y tronco respetando el cuero cabelludo luego se expande, confluye y se transforma en eritema difuso que no afecta las zonas de presión con la ropa; $y$, el periodo de reepitelizacion que puede durar de una a tres semanas dependiendo de la gravedad y extensión de la enfermedad. ${ }^{2}$ El dolor en la piel es un signo temprano destacado y debe alertar sobre una NET que comienza a manifestarse. ${ }^{4}$

El compromiso de órganos internos es inusual y se limita principalmente a las vías respiratorias y tracto gastrointestinal. El daño pulmonar temprano se ve en el $25 \%$ de los casos e incluye tos, obstrucción bronquial y dificultad respiratoria. El compromiso gastrointestinal incluye diarrea, distensión abdominal. El compromiso renal se observa en las fases agudas de la enfermedad y se manifiesta con falla renal aguda, hematuria y microalbuminuria. ${ }^{7}$

Las complicaciones pueden generar morbimortalidad a largo plazo, hay riesgo mayor de sepsis, puede aparecer también despigmentación o cicatrices, también hay distrofias ungueales. Las complicaciones oculares suelen ser las más graves. ${ }^{5,6,8}$

El diagnóstico es clínico, un examen físico completo es esencial para dar un diagnóstico preciso. La biopsia cutánea se usa para confirmar el diagnóstico y se debe realizar siempre. ${ }^{7,8}$
Cualquier medicamento sospechoso debe retirarse de inmediato, así se disminuye el riesgo de muerte. Los casos que ocurren en asociación con procesos malignos o trasplantes de células madres tienen un peor pronóstico y mayor riesgo de muerte. ${ }^{4}$ Dentro del diagnóstico diferencial de SSJ/NET se incluye a las enfermedades bulosas autoinmunes, enfermedades autoinmunes, síndrome de la piel escaldada estafilocócica, erupción farmacológica ampollar fija diseminada. ${ }^{6,9}$

Se requiere un tratamiento multidisciplinario que incluya identificación del fármaco causante, cuidado de la piel y mucosas, la reanimación, el equilibrio de fluidos, un adecuado soporte nutricional, analgesia, monitoreo de infecciones; además, se debe evitar las complicaciones, en especial las infecciones bacterianas. ${ }^{4,9}$

\section{PRESENTACIÓN DEL CASO}

Paciente mujer de 15 años con antecedente de epilepsia con tratamiento habitual fenitoína $100 \mathrm{mg}$ cada 8 horas. Por mala evolución en los últimos meses, se rotó a carbamazepina $200 \mathrm{mg} 2$ tabletas cada 12 horas sin respuesta adecuada. En el último control con su médico se decidió agregar ácido valproico $500 \mathrm{mg}$ cada 12 horas y luego lamotrigina 100 mg cada 24 horas. La paciente acudió a emergencia con historia de fiebre y malestar general, prurito generalizado, cefalea y ardor ocular.

Al examen, PA 90/30 mm Hg, FC 120 latidos/min, FR 19 excursiones $/ \mathrm{min}$, $\mathrm{T} 38{ }^{\circ} \mathrm{C}, \mathrm{SatO}_{2} 94 \%$. Presentaba eritema generalizado, máculas, ampollas en cara, cuello, tronco y extremidades, compromiso ocular (quemosis, secreción amarillenta, visión borrosa), compromiso de mucosa oral y genital (Figuras 1-3). Pulmones, cardiovascular y abdomen sin alteraciones significativas.

Se realizó el tratamiento hemodinámico con hidratación al cual respondió parcialmente, se utilizaron vasopresores. Por mal pronóstico al tener 60 a $80 \%$ de la superficie

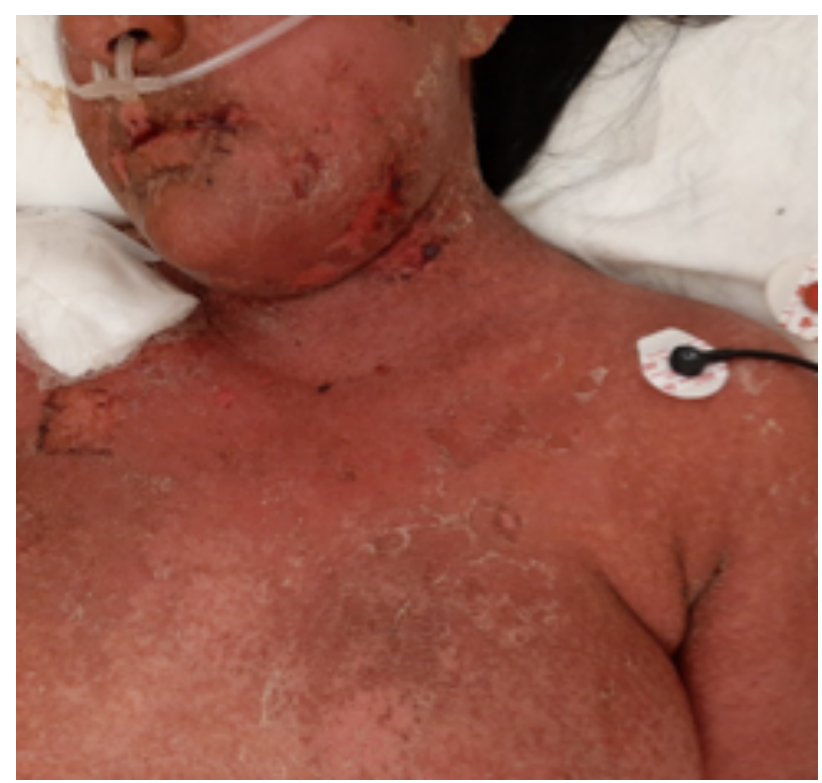

Figura I.Al ingreso. 


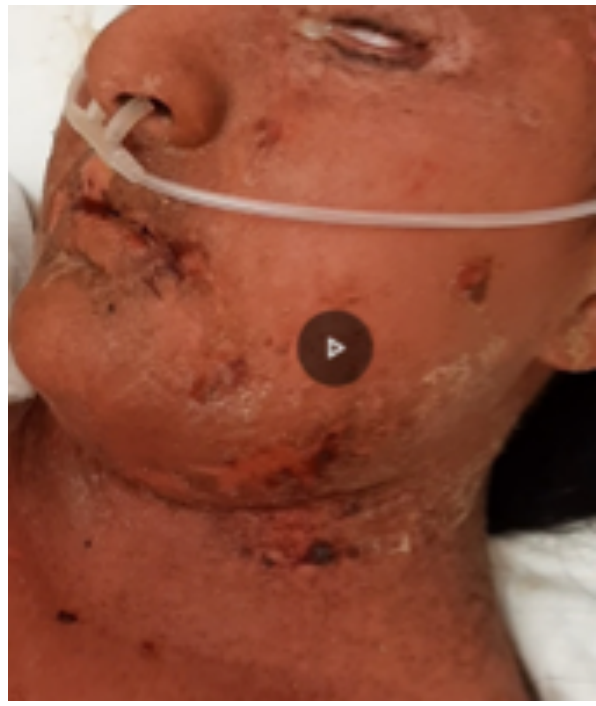

Figura 2.Al ingreso.

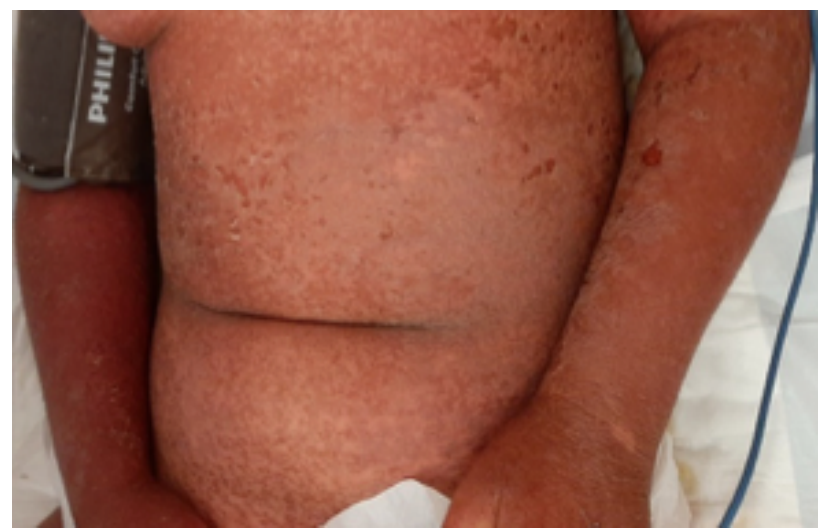

Figura 3.A las 24 horas del ingreso.

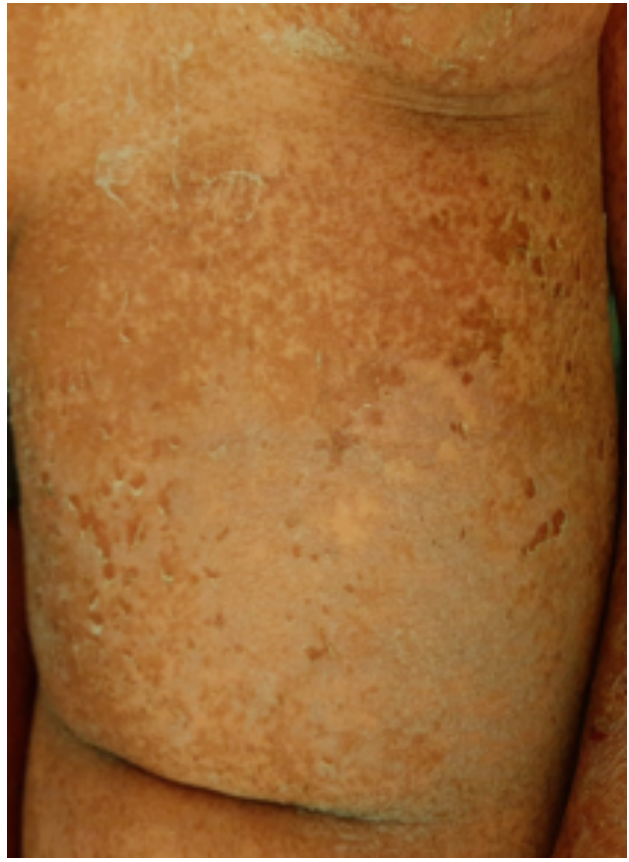

Figura 4.A los 3 días del ingreso.

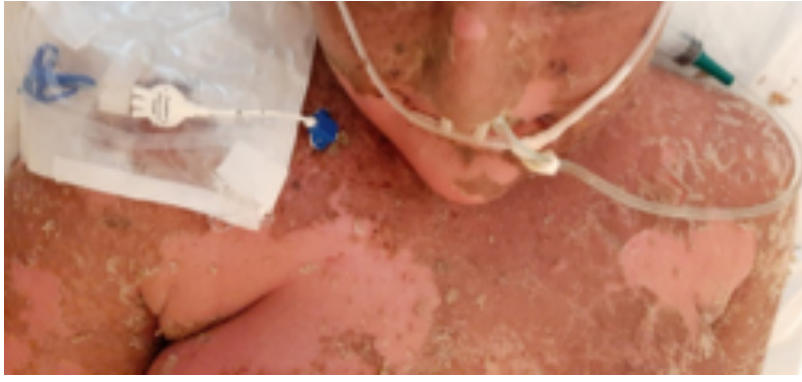

Figura 5.A los 5 días del ingreso.

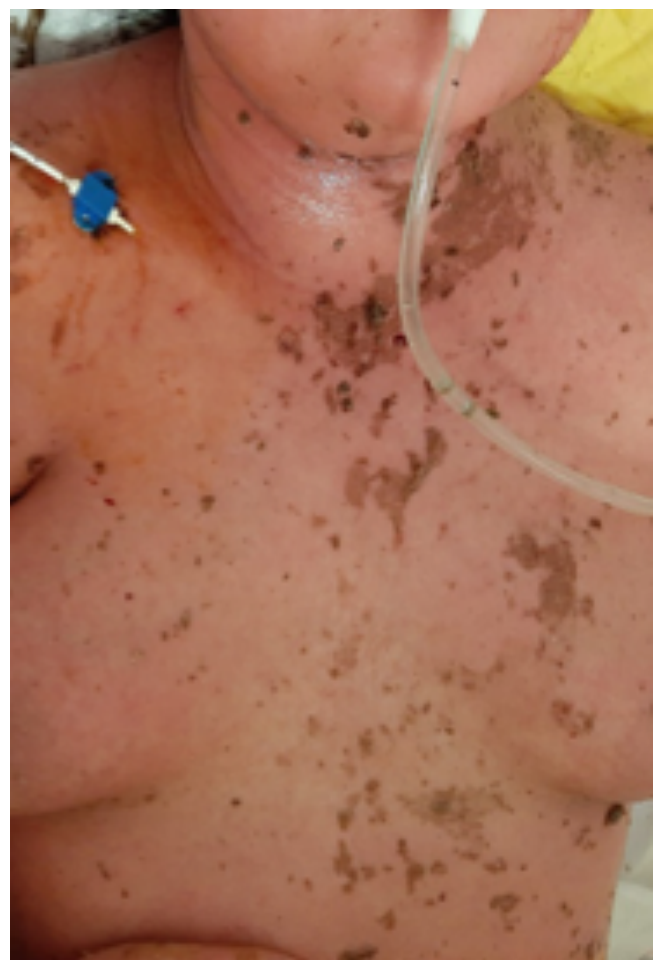

Figura 6.A los 5 días del ingreso.

comprometida y el compromiso hemodinámico y ocular, se sospechó clínicamente de NET con puntuación escala SCORTEN 4, con mortalidad de $64 \%$. Se tomó la biopsia de piel y se decidió iniciar la pulsoterapia precoz (antes de las 24 horas del ingreso) con $1 \mathrm{~g}$ de metilprednisolona por tres días. La mejoría fue notoria desde las 18 horas post pulso, se controló glicemias sin novedad y se realizaron estudios para descartar infecciones subyacentes o concomitantes. A los tres días la mejoría ocular fue notable (Figura 4 y 5), las lesiones de la piel y mucosas a los 4 días post pulso evolucionaron favorablemente (Figura 6).

La biopsia de piel confirmó el diagnóstico de NET.

\section{DISCUSIÓN}

Lo básico en nuestra paciente fue el soporte hemodinámico desde la hidratación, además mantener una temperatura ambiental entre $28{ }^{\circ} \mathrm{C}$ a $32{ }^{\circ} \mathrm{C}$, sobre todo en aquellos pacientes en donde se haya perdido grandes áreas de 
piel. No fue necesario proteger la vía aérea y no hubo necesidad de intubación y ventilación. En estos pacientes el requerimiento de electrolitos y líquidos es menor que para los pacientes con quemaduras de igual extensión. Se indica solución electrolítica y albumina con el fin de mantener el gasto urinario en 0,5 a $1,0 \mathrm{ml} / \mathrm{kg} / \mathrm{h}$. Se indicó nutrición enteral hipercalórica e hiperproteica para evitar la pérdida de proteínas y facilitar la curación. Estos pacientes necesitan menos calorías diarias en comparación con los pacientes quemados. No se recomienda la profilaxis antibiótica. ${ }^{7}$ Los pacientes con NET pierden grandes cantidades de líquidos y electrolitos como sodio, potasio y cloruro. La hipofosfatemia es una complicación común que empeora la resistencia a la insulina y deteriora el estado neurológico y funciones diafragmáticas. Si no se corrige rápidamente se produce deshidratación, la orina se torna hiperosmolar y su producción disminuye, y puede generarse una insuficiencia prerrenal. ${ }^{5}$

La piel afectada se debe limpiar regularmente con agua limpia o solución salina ya que disminuye el riesgo de infección y se recomienda no retirar la piel que se desprende. $^{5}$

Existen algunos reportes que señalan que el uso temprano de corticoides limita la enfermedad y reduce la morbimortalidad; sin embargo, no existen estudios grandes que respalden esto. No hay datos suficientes o estos son contradictorios sobre la eficacia de la terapia sistémica con corticoides en la limitación del daño ocular. Además, existe el riesgo de infecciones secundarias por el uso de corticoides sistémicos y una baja tasa de reepitelización de la piel comprometida, es por ello que se deben administrar con cuidado. Sin embargo, los pulsos tempranos de dexametasona no afectan el tiempo de reepitelización y de estabilización de la enfermedad y no muestran riesgo de sepsis. Se han reportados algunos pocos casos de muertes de pacientes por tratamiento con prednisolona. ${ }^{4,9}$ Otras complicaciones del tratamiento con corticoides son una mayor duración de la hospitalización y una mayor mortalidad. ${ }^{8,10}$ Nuestro equipo tiene experiencia con los pulsos precoces con éxito en el pasado y se decidió, a pesar de no existir evidencia, por falta de estudios en este tipo de paciente, frente a la gran mortalidad, dar los pulsos para salvar la vida.

La dosis es importante porque los estudios realizados no fueron con la dosis de pulso y tampoco con el tiempo precoz. Por ejemplo, en un estudio observacional, retrospectivo, realizado en pacientes, de Alemania y Francia, para evaluar la eficacia de los glucocorticoides no se encontró que estos sean superiores al tratamiento de apoyo, pero solo en cuanto a mortalidad. ${ }^{6,9}$ Otros autores concluyen que el tratamiento con corticoides sistémicos es importante y se deben tener en cuenta como una terapia de bajo costo en países cuyo presupuesto de salud es limitado. ${ }^{9}$ Pero, se ha reportado que los corticosteroides sistémicos son útiles para el control de la enfermedad si estos se administran dentro de los tres días o máximo hasta siete días desde que inician los síntomas. ${ }^{5}$ No usamos inmunoglobulina humana porque su administración no tiene gran impacto, aunque en algunos estudios se señala que ofrecen beneficios en niños. ${ }^{4} \mathrm{Su}$ uso es controvertido y se requieren estudios clínicos más grandes. ${ }^{9}$

Tanto el SSJ como NET son enfermedades con un gran riesgo para la vida del paciente, la mortalidad en el SSJ es del $5 \%$ mientras que para NET es $35 \%$ y depende de la extensión de las lesiones, siendo mayor cuando la lesión ocupa más del $30 \%$ de la superficie corporal. ${ }^{11}$

El fallo cutáneo agudo se caracteriza por una pérdida de integridad de la piel que conduce a un estado catabólico, alteraciones en la inmunidad, riesgo elevado de infecciones, pérdida de la termorregulación, alteraciones hidroelectrolíticas y falla de otros órganos. Es secundaria al daño epidérmico y su gravedad depende de la extensión, puede aparecer cuando una enfermedad específica compromete más del $20 \%$ de la superficie corporal. ${ }^{12}$ Son muchas las causas, siendo algunas de ellas: necrólisis tóxica epidérmica, SSJ, eritema multiforme mayor, eritrodermias, pénfigos, estados penfigoides, entre otras. Es considerado una urgencia dermatológica en donde un diagnóstico precoz y tratamiento adecuado ofrecen mejores posibilidades de recuperación. ${ }^{13}$

Se origina pérdida de agua, electrolitos y calor, lo que hace susceptible a la colonización de la piel y a una infección sistémica secundaria que puede llevar al fallo multiorgánico y la muerte. ${ }^{13}$ Las manifestaciones clínicas sistémicas dependen del grado de compromiso de las funciones de la piel. Causa un deterioro progresivo del estado general y puede haber edema, deshidratación, taquicardia, dificultad respiratoria. ${ }^{14}$ Además, por la inmovilización prolongada se puede desarrollar trombosis venosa profunda. ${ }^{15}$ El manejo requiere trabajo en equipo, los pilares son: un adecuado monitoreo de cambios hemodinámicos; balance de líquidos, electrolitos y nutrición; y, la prevención de complicaciones. ${ }^{15}$

En conclusión, se presentó un caso de NET que fue tratado exitosamente con pulsoterapia de metilprednisolona, quedando pendiente obtener mayor evidencia con estudios controlados.

\section{REFERENCIAS BIBLIOGRÁFICAS}

1. Fernández Carmona A, Redondo A, Olivencia-Peña L, Gómez A, Pareja J, Martínez J. Tratamiento de necrólisis epidérmica tóxica con ciclosporina A. Med Intensiva. 2011; 35(7): 442-445.

2. Estrella-Alonso A, Aramburu JA, González-Ruiz MY, Cachafeiro L, Sánchez Manuel S, Lorente JA. Necrólisis epidérmica tóxica: un paradigma de enfermedad crítica. Rev. bras. ter. intensiva. 2017; 29 (4): 499-508.

3. Fernández FA, Pintor E, Quesada R, Garcés FJ. Necrólisis epidérmica tóxica inducida por fenitoína y radioterapia holocraneal. Actas Dermosifiliogr. 2007; 98:483-5.

4. McPherson T, Exton LS, Biswas S, Creamer D, Dziewulski P, Newell L, et al. BAD guidelines for SJS/TEN in children and young people, 2018. BJD. 2019; 181: 37-54.

5. Gupta LK, Martin AM, Agarwal N, et al. Guidelines for the management of Stevens-Johnson syndrome/toxic epidermal necrolysis: An Indian perspective. Indian $J$ Dermatol Venereol Leprol. 2016; 82:603-25

6. Harr T, French L. Toxic epidermal necrolysis and Stevens-Johnson syndrome. Orphanet J Rare Dis. 2010; 16(5): 39. 
7. Dodiuk-Gad RP, Chung WH, Valeyrie-Allanore L, Shear NH. Stevens-Johnson syndrome and toxic epidermal necrolysis: An update. Am J Clin Dermatol. 2015; 16(6):475-93.

8. Mockenhaupt M. Stevens-Johnson syndrome and toxic epidermal necrolysis: clinical patterns, diagnostic considerations, etiology, and therapeutic management. Semin Cutan Med Surg. 2014; 33(1):10-6

9. Lerch M, Mainetti, C, Terziroli Beretta-Piccoli B. et al. Current perspectives on Stevens-Johnson syndrome and toxic epidermal necrolysis. Clin Rev Allergy Immunol. 2018;54(1):147-176.

10. Schwartz R., McDonough P. Lee B. Toxic epidermal necrolysis: Part II. Prognosis, sequelae, diagnosis, differential diagnosis, prevention, and treatment. J Am Acad Dermatol. 2013; 69(2):187.e1-e16

11. Sotelo-Cruz N. Síndrome de Stevens-Johnson y necrólisis epidérmica tóxica en los niños. Gaceta Médica de México. 2012; 148: 265-75.

12. Blanco GM, Abascal JM, Cortés OM, Matos FN. Fallo cutáneo agudo por síndrome de Steven Johnson. A propósito de un caso. IntraMed Journal. 2016;5(3).

13. Mora Castillo NE, Hernández Del Pino S, Aroche Domínguez ME, Pérez Mora ND. Fallo multifuncional cutáneo agudo. Actualización. Rev. inf. cient. 2010; 65(1).
14. Sánchez L, Pancorro J, et al. Insuficiencia cutánea aguda. Dermatol. peru. 2002; 12 (3): 175-182.

15. Inamadar AC, Palit A. Acute skin failure: Concept, causes, consequences and care. Indian J Dermatol Venereol Leprol. 2005; 71:379-85.

Conflicto de intereses:

Los autores declaran no tener conflicto de intereses.

Financiamiento:

Autofinanciado. Autor

Corresponsal:

Stalin Vílchez Rivera. Dirección: Libertad 556 - Santa Victoria.

Chiclayo - Perú.Teléfono: 051914138409.

\section{CORRESPONDENCIA:}

sdelarivera@hotmail.com

Fecha de recepción: 30-04-2020.

Fecha de aceptación: 25-05-2020. 